\title{
Samuel Colt, une légende américaine
}

\section{Samuel Colt, an American legend}

\author{
Daniel Casanova ${ }^{1}$ \\ ${ }^{1}$ auteur indépendant, France
}

RÉSUMÉ. Samuel Colt est un personnage hors normes. Aventurier, passionné de mécanique et de chimie, il va se révéler comme un véritable entrepreneur d'industrie. A la fois bonimenteur et séducteur, tout au long de sa vie, il va développer son invention : le revolver et pour cela devenir un véritable homme d'affaires. II s'assure des protections juridiques pour ses brevets, profite du contexte historique (conquête de l'Ouest, guerre avec le Mexique puis guerre de Sécession), augmente la productivité de sa fabrique et fait preuve de clairvoyance en matière de publicité et de marketing promotionnel. Samuel Colt sait se servir de son entregent pour offrir des armes de luxe aux hommes clef de différents états. II sait rationaliser son usine, conçue selon ses plans, appliquant une sorte de segmentation du travail avant la lettre et appliquer un paternalisme social pour se fidéliser ses ouvriers.

ABSTRACT. Samuel Colt was an extraordinary character. Adventurer, passionate about mechanics and chemistry, he would show himself to be a true entrepreneur of industry. Both a good talker and a seducer, throughout his life, he would develop his invention: the revolver, and for that he would become a real businessman. He secured legal protection for his patents, took advantage of the historical context (conquest of the West, war with Mexico and also the Civil War), increased the productivity of his factory and showed foresight in advertising and promotional marketing. Samuel Colt knew how to use his interpersonal skills to offer luxury weapons to key men in different states. He knew how to rationalize his factory, designed according to his plans, applying a kind of segmentation of work before this had really been conceived and also applied a social paternalism to gain the loyalty of his workers.

MOTS-CLÉS. Colt, entrepreneur, armement, contexte historique, Etats-Unis.

KEYWORDS. Colt, entrepreneur, armament, historical context, USA.

\section{Introduction}

Samuel Colt (fig. 1) nait le 19 juillet 1814 à Hartford dans le Connecticut, aux États-Unis, dans une famille de six enfants. Dans son enfance, il connait parfois des jours difficiles car les affaires de son père, qui possède une filature de soie, ne sont pas toujours florissantes. Il reçoit une bonne éducation à l'Académie d'Amherst, où il se passionne pour les sciences mécaniques et la chimie. Il obtient un équivalent du brevet élémentaire.

En 1829, il invente une mine sous-marine qui explose parfaitement au fond d'un étang mais arrose de boue tous les spectateurs de la ville de Ware. Après cet «exploit », son père envoie, son jeune turbulent de fils, en qualité de matelot, en Europe et aux Indes entre 1830 et 1831 . Il embarque à Boston sur un Brick le «Corvo » à destination de Calcutta. La légende veut que l'idée d'un revolver moderne, car le système était connu depuis le début des armes à feu, lui soit apparue sur le bateau en observant le timonier. Ce dernier tourne et bloque dans la bonne position la roue qui commande le gouvernail ! Il aurait trouvé là, l'idée du verrouillage du barillet, problème sur lequel se heurte beaucoup d'armuriers. Notre jeune marin sculpte un prototype en bois qu'il présente, à son retour, à son père et à un ami de la famille Henry L. Ellsworth membre de la Commission des brevets américaine. Les deux hommes lui conseillent de perfectionner son invention et de déposer un brevet pour la protéger.

Pour financer ses recherches, Samuel se fait bateleur. Sous le pseudonyme de « Docteur Coult de Calcutta » il fait des conférences sur les gaz hilarants à travers les Etats-Unis et le Canada. Moyennant finance, il teste son gaz sur les volontaires! Le produit de ses tournées est en grande partie versé à des armuriers de Hartford, Albany et Baltimore qui, entre 1832 et 1836, réalisent les prototypes de ses inventions. Des revolvers, certes, mais aussi des carabines-revolvers et un fusil de chasse ! Le «docteur Coult»s'adonne à la réclame, aux relations publiques et à l'art oratoire qui 
font du jeune Colt un publicitaire consommé. Il cajole ainsi ses armuriers pour la réalisation de ses armes qui fonctionnent plus ou moins bien. Jon Pearson de Baltimore réalise enfin un prototype de revolver fonctionnel vers 1835. C'est le seul professionnel qui comprend réellement les idées du jeune Samuel.

Très peu d'études académiques sont disponibles sur les fabricants d'armes ${ }^{1}$. Dans le cas de Samuel Colt, comme pour beaucoup d'autres, les publications sont souvent l'apanage de collectionneurs qui fouillent les archives familiales, étudient les objets et les brevets et, avec quelques musées, préservent ce patrimoine historique. En Europe, les usines ayant été bombardées lors de différentes guerres ou pillées les archives ont presque toutes disparu. Aux Etats-Unis, il s'agit surtout de faillites ou de rachat qui faisait que l'inventeur quittait l'entreprise avec ses archives. Les études pour les Etats-Unis proviennent essentiellement de la National Rifle Association (NRA) et de la American Society of Arms Collectors (ASAC).

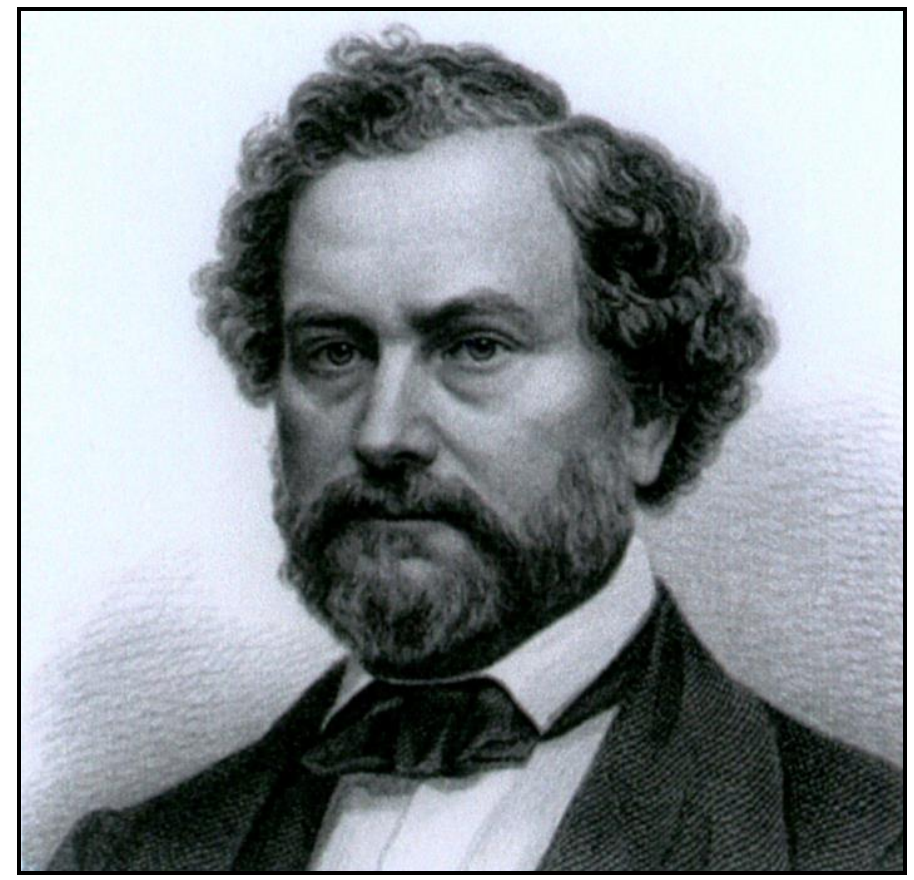

Figure 1. Portrait de Samuel Colt

Source : catalogue Armsmear, 1866 (libre de droit).

\section{Une entreprise américaine bâtie sur une gamme d'innovations}

\subsection{Un revolver moderne}

Les utilisateurs d'armes de poing rêvaient d'armes à répétition. La première idée est de doter les pistolets de plusieurs canons. Mais ces armes multi-canonnées sont lourdes et encombrantes. Elles sont surnommées les poivrières ! $\mathrm{Au} \mathrm{XIV}^{\mathrm{e}}$ siècle, apparaissent les bombardes et autres canons. Très vite, on invente le canon à main, ou couleuvrine, que l'on nomme aussi bâton de feu. Il s'agit d'un canon percé au tonnerre d'un trou appelé lumière, reposant sur une crosse en bois rudimentaire. Par la bouche, le soldat place la poudre puis une bourre et la balle. Il force légèrement le tout avec une baguette. Il place alors le boutefeu sur la lumière, en fait une mèche incandescente. La poudre s'enflamme et détonne, lançant la balle. Il faut, pour se servir de ce canon à main, deux hommes : un pour le tenir, l'autre pour manier le boutefeu. Si le tireur est seul, le canon doit reposer sur un support. Les premiers armuriers créent un système de mise feu qui s'appelle la platine à mèche. La mèche, en chanvre de lin mêlé de salpêtre, est portée à incandescence. Elle se place dans les mâchoires d'une pièce de métal en forme de $\mathrm{S}$, mue par un ressort. Le tireur presse la partie 
inférieure du $\mathrm{S}$ et la partie supérieure qui tient la mèche vient se plaquer sur la lumière. L'arquebuse est née. Elle repose sur une fourche (nommée fourquine) dans un premier temps. Pour faciliter la mise à feu, on crée sur la lumière un bassinet, rempli de poudre plus fine, qui restera en service jusqu'au XIX ${ }^{\mathrm{e}}$ siècle. Il existe quelques pistolets à mèche, notamment au Japon, mais l'ensemble est peu pratique. Le Musée de l'armée à Paris comme celui de l'arsenal de Venise possèdent un mousquet à mèche doté d'un barillet ! L'idée est audacieuse. Le soldat place une charge dans chaque chambre du barillet. Il tourne celui-ci à la main pour placer une chambre chargée devant le canon. Il presse ensuite la détente qui place la mèche incandescente dans le bassinet dont sont pourvues chaque chambre du barillet. L'opération est dangereuse car le risque d'inflammation de toutes les charges est bien réel. De plus, l’opération est longue car il lui faut ré-enflammer la mèche. L'arme de Paris possède qu'un seul bassinet et provient du cabinet de Louis XIII. Il s'agit d'une œuvre de maitrise d'arquebuserie plutôt que d'une arme pratique. Cette mèche enflammée reste une source de problèmes par temps humide ou par grands vents. Elle doit rester allumée à un bout. Le mousquetaire s'en sert pour ré-enflammer l'autre bout, celui en contact avec la lumière qui s'éteint au départ du coup [CAS 14].

L'horlogerie vient au secours de l'armurerie, vers 1530 , avec l'invention de la platine à rouet qui comporte une roue dentée devant la lumière. Une clef permet de remonter le ressort qui fait tourner le rouet. Le levier, appelé chien, enserre non plus une mèche mais un morceau de pyrite. Le mécanisme de détente libère le ressort et la roue frotte sur la pyrite. La gerbe d'étincelles enflamme la poudre et le coup part. Vers la même époque apparaissent les premiers canons rayés, qui donnent un mouvement gyroscopique stabilisateur à la balle de plomb. La précision s'accroit, mais il faut forcer la balle dans les rayures, à l'aide d'un maillet et d'une baguette de fer. Ces armes sont coûteuses et seuls les nobles et les corps d'élites, comme la cavalerie, en sont dotés. Les armées restent fidèles au mousquet à mèche pour cette question de coût et pour la rapidité de chargement.

Les pistolets à rouet à deux canons superposés sont rapidement introduits. Ces armes possèdent deux platines. Elles sont lourdes et peu pratiques. Il existe au musée de l'armée de Prague une carabine revolver à rouet, très lourde et très richement gravée, commandée, sans doute, par un noble amateur fortuné. Il faut attendre l'arrivée de la platine à silex, beaucoup plus légère pour que les armuriers repensent à l'idée d'un barillet ! La platine va évoluer jusqu'au début du XVII ${ }^{\mathrm{e}}$ siècle pour se stabiliser sous la forme de platine à silex à la française. Le chien enserre un morceau de silex taillé en biseau. Le tireur arme le chien en le tirant en arrière, comprimant un ressort interne, puis il presse la détente qui actionne une gâchette libérant le ressort. Le chien part en avant et le silex frappe la batterie (plaque de fer cémentée). Sous le choc, la batterie se soulève, découvrant le bassinet rempli de poudre et placé devant la lumière. Le choc du silex sur le fer provoque des étincelles qui tombent dans le bassinet et enflamment la poudre d'amorce, qui, à travers la lumière, fait détonner la charge de poudre contenue dans le canon. La platine à silex «à la française » est la plus élaborée. Elle est la synthèse de deux autres systèmes le chenapan et le miquelet. La platine à chenapan s'est développée, vers 1560, en Hollande et en Scandinavie, la batterie ne protège pas le bassinet. Dans les pays du sud, Espagne et Italie, on rencontre la platine à la Miquelet aux solides ressorts extérieurs. Les cavaliers utilisent les pistolets par paire, mais leur puissance de feu reste faible. Très vite, les armuriers proposent des pistolets à plusieurs coups. Tout d'abord des pistolets à deux coups à canons en table ou superposés. Viennent ensuite les armes à canons tournants deux, trois voire quatre ! Sur chaque canon se trouve un bassinet et sa batterie. Les canons se tournent à la main. Ces armes se rencontrent régulièrement dans les musées et les collections privées, preuve de leur succès. Cela reste des armes lourdes et encombrantes. Aussi certains armuriers français ou anglais repensent au revolver. Ils montent alors un barillet classique de trois à quatre chambres que l'on tourne à la main. Ces premiers revolvers sont magnifiques, car destinés à un public fortuné de connaisseurs. Ils sont conçus à l'unité. Sur chaque chambre du barillet se trouve la batterie et le bassinet. La platine et le chien sont solidaires de la crosse. Le problème reste l'étanchéité peu parfaite entre le canon et le barillet. L'arme est peu pratique à transporter avec les plaques de batterie qui forme des protubérances. D'où l'idée de revenir à une seule batterie. Naturellement, ceci 
implique qu'il faudra recharger chaque fois le bassinet. Le gain de temps reste appréciable car les canons sont de leur côté déjà chargés ! Sur cette idée, l'américain Elisha Haydon Collier (17881856), de Boston, conçoit un revolver rationnel, le premier de l'histoire de l'armement, vers 1814 . Il brevette son idée, en 1818, à Londres. Le barillet tourne avec l'armement du chien et un compartiment de poudre s'ouvre en même temps pour déverser cette dernière dans le bassinet. Cependant, lors de la mise en fabrication, E. H. Collier abandonne la rotation automatique du barillet, trop complexe, qui se fait à la main. C'est John Evans \& Son, à Londres, qui assure la fabrication. Entre 1819 et 1824 plus de dix mille pièces sont produites, dont une large partie est envoyée aux Indes où Samuel Colt a dû en voir. Entre-temps ces revolvers utilisent la nouvelle platine à percussion! Le bassinet disparait, remplacé par une cheminée que vient coiffer une amorce. Le chien affecte alors la forme d'un marteau pour venir percuter l'amorce.

Samuel Colt modernise et rationalise le concept du revolver : un pistolet doté d'une chambre rotative à plusieurs coups appelée barillet. A Londres, lors de l'escale de son bateau, il peut voir chez les armuriers anglais le revolver Collier qui fonctionne avec un système de mise à feu à silex, transformé à percussion. Le nom de cette arme vient du verbe anglais to revolve (tourner). Ce nom sera repris en français. Mais le système Colt est plus ingénieux : il bénéficie de l'invention du système de mise à feu à percussion composé d'une capsule détonante (ou amorce) et d'une balle ; d'où l'appellation anglaise caps and balls. L'armement du chien provoque la rotation du barillet et l'apparition de la détente. Ce mécanisme, en simple action, est fiable. Une pression sur la détente libère le chien qui s'abat sur l'amorce de la chambre du barillet, placée devant le canon. Le coup part. C'est simple et direct et autorise un tir précis. Le montage du canon, via une clavette, sur l'axe du barillet est assez solide pour supporter de fortes charges. Il permet aussi un démontage pratique du barillet pour le recharger. Plus tard, il va concevoir un levier refouloir sous le canon pour recharger l'arme sans la démonter. Suivant les conseils de son père et de Henry Ellsworth, il se rend personnellement en France ${ }^{2}$ et en Angleterre pour déposer un brevet en 1835. L'année suivante, il fait de même aux Etats-Unis ${ }^{3}$.

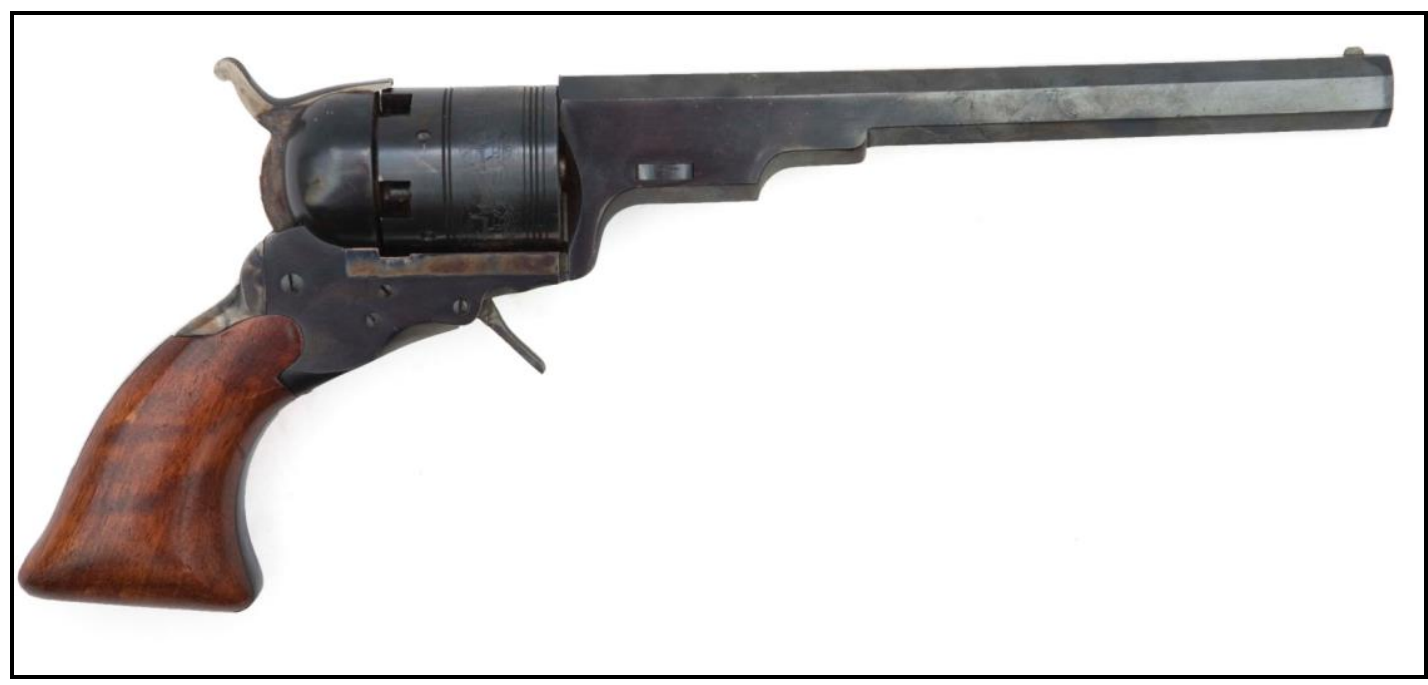

Figure 2. revolver Colt Paterson

Source : photographie de l'auteur

\subsection{La société PATENT ARMS MANUFACTURING COMPANY}

Fort de son expérience de bateleur Samuel Colt présente, dès 1835, son prototype dans les Etats de New York et du New Jersey pour recruter des actionnaires. Il peut ainsi, pour exploiter son

\footnotetext{
${ }^{2}$ INPI dossier 1BA5164, brevet déposé le 16 novembre 1835

${ }^{3}$ US Patent 9430 du 25 février 1836 
brevet, créer la Patent Arms Manufacturing Company à Paterson, dans le New Jersey. Trop jeune, 22 ans, pour en devenir le Président, aux yeux des actionnaires, il se réserve le poste de vendeur démonstrateur et d'ingénieur conseil. Naturellement en plus de son salaire annuel de 1000 dollars, il reçoit 6000 dollars pour démarrer et un pourcentage sur les ventes. En juin 1836, Colt commence ses tournées, il fait déjà du marketing et traite somptueusement les fonctionnaires pouvant lui concrétiser des marchés. Les Etats-Unis se sont construits dans la violence. Principalement contre les Indiens, puis les Français du Canada et enfin contre les Anglais pendant la guerre d'Indépendance. Cet état d'insécurité conduit chaque homme à être armé. On trouve des armes d'importation françaises et anglaises puis des fabrications locales de plus en plus importantes, dues aux difficultés des voyages en mer lors des guerres napoléoniennes. Les armuriers américains vont ainsi créer pour certains de véritables dynasties comme Remington et Deringer puis Colt ainsi que Smith \& Wesson. Un des enjeux de ces armements est les milices locales, très importantes dans les colonies américaines. La société Colt en équipera de nombreuses depuis les Texas Rangers aux miliciens du Potomac.

Mais les armes rencontrent des défauts de fonctionnement et leurs prix sont élevés. Ces revolvers sont fabriqués à près de deux mille huit cent exemplaires, entre 1836 et 1842 , dans différents calibres et longueurs de canons. Auxquels s'ajoutent quelques carabines revolvers. Son meilleur client est l'Etat du Texas qui lui en achète 180 pour les Texas Rangers.

«Sous la direction du capitaine John Coffee «Jack» Hays, les Rangers, nouvelle police de la République du Texas repoussent les attaques des Indiens et des Mexicains. Hays impose une stricte discipline et un esprit de corps. L'un de ses officiers, le capitaine Samuel Hamilton Walker lui suggère d'adopter le revolver Colt. L'arme fait merveille, notamment à la bataille de San Gabriels (15 au 17 mai 1839) où les Texas Rangers mettent en déroute une bande d'Indiens et de Mexicains. Ces derniers, surpris par la puissance de feu des Rangers, se font abattre avant d'avoir pu recharger leurs propres pistolets à un coup et ne peuvent pas utiliser leurs lances. » [IVE 10]

Cependant l'arme est un échec du point de vue commercial, à cause de nombreuses difficultés financières et la perte de confiance des actionnaires. En 1842, la société est liquidée. Mais Samuel Colt ne se décourage pas et croit à l'avenir de son invention. D'autant que son brevet couvre son invention jusqu'en 1857.

\subsection{Les revolvers Walker et Dragoon}

La guerre contre le Mexique, en 1846, propulse des officiers expérimentés comme Hays et Walker sur le devant de la scène. Ce dernier, fort de son expérience avec les Texas Rangers, demande à Washington des revolvers Colt pour équiper ses dragons! Samuel Colt réagit très vite et fait construire un revolver amélioré avec l'aide de Walker. L'armée lui commande mille exemplaires. Il passe un contrat avec Eli Whitney Jr qui possède l'usine adéquate pour construire ce gros revolver de calibre 44 (soit 44 centièmes de pouce ou $11 \mathrm{~mm}$ ), de plus de deux kilos ! Colt le nomme «Walker » en l'honneur du capitaine, tué par les Mexicains, le 9 octobre 1847, et le barillet porte gravé le dessin de la bataille de Bandera Pass où Hays s'illustra avec ses Rangers. [SIB 78].

«En 1841, Hays à la tête de cinquante Rangers sont attaqués par une tribu de Comanches. Les Rangers chargent revolver au poing. Les "colt» déciment les Indiens surpris par la cohésion des rangers et les nouvelles armes inconnues... » [IVE 10]

Le Walker possède un pontet et un levier refouloir, il préfigure la silhouette de tous ses successeurs. Fin 1847, Samuel Colt investit ses bénéfices dans une nouvelle usine située à Hartford. Il construit des revolvers baptisés «Withneyville Dragoon » à partir des pièces détachées des Walker. Il s'agit d'une nouvelle amélioration pour corriger les défauts du Walker (éclatement des canons ou des barillets). Puis avec le produit des ventes de ces revolvers de transition, il crée un nouveau revolver le «Dragoon » qui sera fabriqué à vingt-deux mille exemplaires. Il en existe trois 
versions dont la dernière possède une crosse d'épaulement. L'arme se vend surtout sur le marché civil, pionniers, cowboys, sheriffs et miliciens, la petite armée fédérale étant forte que de 16000 hommes ne lui en prend que 300. Pour répondre à cette demande civile, Colt sort, en 1848, une version « Pocket ». Cette version réduite, appelée «Baby Dragoon » connait un rapide succès et est améliorée en 1849 et 1850. Ce dernier modèle étant adopté par la Wells Fargo, pour équiper les convoyeurs des diligences, sans levier refouloir. Il va faire partie de la légende du Far West.

En commercial avisé, Samuel Colt offre des paires de revolvers à des officiers généraux comme Franklin Pierce et Zachary Taylor faisant partie du bureau du Président des Etats-Unis ou encore au général Jefferson Davis qui sera élu Président de la Confédération sudiste en 1861. Il obtient ainsi de nombreux petits contrats militaires. Pour se protéger des contrefaçons et des imitateurs, il fait graver les numéros de ses brevets sur tous ses revolvers et charge ses avocats de poursuites judiciaires, jusqu'en Belgique. Liège est en effet un grand centre armurier, le plus important d'Europe, devant Saint-Etienne et Birmingham. Les armuriers belges sont des spécialistes de la copie des armes à succès. Ils déposent forces brevets, modifiant légèrement les inventions, pour se protéger des foudres juridiques, voire même signant Colt Breveté, sans payer les royalties (10 francs belges). Une pratique qui va durer jusqu'en 1914. Le représentant légal de Colt est Monsieur DevosSera qui se charge de confisquer les revolvers contrevenant lors de leur passage au banc d'épreuve de Liège.

\subsection{Les revolvers 1851}

Tous les revolvers Dragoon à six coups sont au calibre $44(11 \mathrm{~mm})$ puissant mais lourd. En 1851, Colt présente un nouveau revolver en calibre $36(9 \mathrm{~mm})$ baptisé « Navy ». Le barillet porte gravée la bataille navale de Campêche, qui vit s'affronter, le 30 avril 1843, la flotte texane à une escadre mexicaine. Le succès est immense, 250000 exemplaires fabriqués, c'est vraiment l'arme de la conquête de l'ouest. Plus présente que les autres modèles de la marque [SEL 71]. Le Colt Navy 1851 fut utilisé par les aventuriers comme Wild Bill Hickok, John Henry « Doc » Holliday, les généraux sudistes comme Robert E. Lee et Nathan B. Forrest, ou les raiders sudistes de la «bande à Quantrill ». C'est l'une des armes standard de la guerre de sécession. L'arme est d'ailleurs copiée au Nord comme au Sud. Le Colt Navy par ses lignes épurées rend obsolètes les anciens «Dragoon». Finalement l'US Navy va en acheter mille exemplaires!

En 1849, Colt effectue son troisième voyage en Europe pour s'assurer de la protection de ses brevets et accorder des licences de fabrication à des armuriers anglais et belges. Il possède la fabrique d'armes privée la plus rentable, la mieux organisée et la plus vantée dans les journaux. Colt achète un entrepôt avec ses seuls bénéfices sur les marchés d'Etat. Il s'entoure d'une équipe d'amis. Edward Dickerson, son conseil en brevet, se charge de protéger et prolonger celui-ci jusqu'en 1857. Elisha K Root, ingénieur, organise la production. William Tuller contrôle celle-ci. Joslin et Sargeant gèrent les finances. Samuel n'hésite pas à se séparer de son frère James B Colt qui est le maillon faible. La fabrique compte 300 ouvriers. Colt adapte au mieux ses locaux mais pense déjà plus grand ! Il se fait nommer lieutenant-colonel de la Milice du Connecticut en 1850. Il va ainsi utiliser son titre pour mieux séduire les militaires du monde entier. A Hartford tout le monde le nomme colonel Colt ! Sa gamme de revolver couvre tous les besoins avec les Pocket 1849 en calibre 31 (7 $\mathrm{mm})$, Navy en calibre $36(9 \mathrm{~mm})$ et Dragoon en calibre $44(11 \mathrm{~mm})$. Aux vues de sa situation de monopole, ces calibres vont devenir des références pour ses concurrents et même en Europe.

\section{Le développement international de l'entreprise Colt}

\subsection{La création d'une usine Iondonienne}

Samuel Colt aime beaucoup l'Angleterre et il va s'y rendre une demi-douzaine de fois à partir de 1850. Lors de l'Exposition Universelle de Londres en 1851, son stand est le plus beau du pavillon 
américain. De nombreux officiers peuvent y manipuler ses créations. Il est présenté à la Reine Victoria. Naturellement, en homme d'affaire avisé, il se réserve une part des 450 revolvers qu'il a emmenés. Il offre ainsi de somptueux coffrets au prince consort Albert et au prince de Galles Edouard. Mais aussi à de nombreux dignitaires britanniques à la fin de l'exposition.

Colt implante son usine anglaise, en 1852, à Londres au bord de la Tamise. L'usine équipée de machines-outils américaines et contrôlée au début par des armuriers formés à Hartford produit des revolvers de qualité. Il se fonde sur le principe du « self made man », un principe qu'il s'est appliqué à lui-même. Il possède des idées en avance sur son temps et applique une segmentation du travail ; une rationalisation qui sera appliquée plus tard par Henry Ford dans l'automobile. Samuel Colt va appliquer son mode de gestion en grand aux Etats-Unis dans sa nouvelle manufacture sans jamais perdre de vue ses intérêts. Les perspectives d'avancements qu'il offre, lui attache ses ouvriers. Avec la guerre de Crimée (1854-1856) les ventes montent en flèche (23 000 revolvers Navy commandés par l'Armée britannique, 700 Dragons, 11000 modèles Pocket et 42000 Navy sur le marché privé). Mais à partir de 1857, la concurrence anglaise devient trop forte et l'usine doit fermer.

En Angleterre, les brevets Colt sont contournés par un nouveau mécanisme. Robert Adams présente, en 1851, son premier revolver à la grande exposition qui se tient au Cristal Palace, dans Hyde Park, à Londres. Il se présente comme le concurrent direct de la société Colt. Son revolver, fabriqué par la firme Deane père et fils, est à double action seule, contrairement au revolver américain en simple action. C'est le début d'une polémique entre les deux systèmes, le premier offrant une grande rapidité de tir et le second une meilleure précision. Cependant la guerre de Crimée met en lumière les faiblesses de l'arme anglaise face au Colt ; le manque de précision et l'absence de levier refouloir. Arguments repris par les agents de Colt à Londres. Sur l'Adams, les chambres du barillet légèrement plus larges et tronconiques n'arrivent pas à maintenir solidement les balles enfoncées avec le pouce. Un handicap très sérieux pour un cavalier ! Le lieutenant Frédéric Beaumont, officier du Génie, met fin à la polémique engagée entre les deux firmes par l'invention d'une platine double et simple action, le 20 février 1855. Le brevet s'applique sur le revolver Adams. Le Board of Ordnance comprend immédiatement l'intérêt du brevet et commande cent pièces pour mars, puis deux mille pour la fin de l'année.

La Chambre des Communes s'émeut en 1854 de l'influence de l'usine Colt sur l'industrie d'armement anglaise. Le Lieutenant-Colonel Sam Colt est emmené à y déposer. Nous extrayons de sa déposition qui fait dix-sept pages, quelques questions [HAV 40]:

La 1084 : "Vos pistolets sont-ils fabriqués essentiellement par des machines ? Entièrement sauf l'ébarbage et le transfert des pièces d'une machine à l'autre, la finition et l'assemblage. »

La 1085 : "Estimez-vous que vos pistolets soient mieux fabriqués par des machines qu ils ne pourraient l'être à la main? Absolument!»

\section{La 1086 : "Et moins cher? Beaucoup moins cher!»}

La 1152 : "Avez-vous eu beaucoup de difficultés avec vos employés ici au début ? J'ai eu pas mal d'ennuis au début. J'ai rencontré d'énormes difficultés; j'avais d'abord fait venir quelques Américains pour aider au démarrage comme contremaitres, mais le climat et les habitudes des gens ne leur ont pas convenu et ils sont partis. J'ai alors commencé à embaucher les ouvriers les plus qualifiés que je pouvais trouver pour exécuter différentes tâches, mais j ai dî me séparer de tous. Ensuite, j'ai essayé les moins qualifiés et les moins chers que j ai pu trouver, et plus l'homme était ignorant plus il était intelligent pour ce que je voulais; ;e résultat a été le suivant; j a ri maintenant des hommes à mon service que j'ai commencé à payer deux shillings par jour et, en l'espace d'un an, je n'en ai pas de trop à huit shillings par jour; il y en a quelques-uns à qui je paie ce salaire et je ne les laisserais pas partir maintenant sils me demandaient neuf shilling. Voilà ce que l'on obtient lorsqu'on emploie des gens qui ne savaient rien au départ. Ils commencent d'abord comme 
manœuvres à deux shillings par jour, puis, si je les trouve capables et honnêtes, je les emploie comme gardiens ou pour peser le métal. Très vite, si un poste devient vacant à une machine, je les y affecte. Ils passent alors de deux shillings au cours des premiers mois à quatre ou cinq shillings et petit à petit, ils deviennent des ouvriers qualifiés...Les meilleurs sont payés huit shillings par jour et finissent par devenir contremaitres. Ne m'emmenait pas quelqu 'un qui sait quelque chose si vous voulez que je lui apprenne quelque chose. »

La 1153 : question posée par Mr S Walpole (Ministre de l'Intérieur) : "Vous voulez des gens intelligents mais de peu de savoir? Oui, je prends la matière brute... »

La 1171: "Vous avez été incité à ouvrir votre établissement afin d'amener le travail à la perfection et d'en tirer les plus grands bénéfices possibles? Oui, et aujourd'hui je suis fier du résultat de mes efforts, je peux mener ma propre barque... »

L'homme est direct, immodeste et sûr de lui. Il possède une conception d'une entreprise qui lui est propre et prend soins à sa manière de ses employés, en leur offrant des opportunités de carrières. Une magnifique paire de Colt Navy fabriquée à Londres est offerte à sa Majesté Napoléon III. L'inventeur écrit une longue lettre en 1854: «Le Col. C. se permet de rappeler à Sa Majesté l'occasion au cours de laquelle Elle a essayé les revolvers Colt à Saint-Cloud. Le Col. Colt ayant eu l'honneur d'être présenté par son Altesse le prince Murat». La lettre propose les services de sa société et même de créer un dépôt en France. Napoléon III se heurte malheureusement au conservatisme des officiers supérieurs. L'armée française n'adoptera un revolver français qu'en 1870 mais la Marine Impériale dès 1858. Il s'agit aussi de revolvers français fabriqués par Eugène Lefaucheux. En 1855, la même démarche auprès du Tsar aboutie à une commande de 5000 pièces et l'autorisation pour l'arsenal de Toula de fabriquer des copies.

Casimir Lefaucheux présente, en 1851, à l'exposition de Londres, au Cristal Palace, une poivrière qui tire des cartouches à broche, en carton. Depuis 1835, l'armurier parisien détient le brevet de cette cartouche qui a l'avantage d'être étanche et connait un grand succès au niveau des fusils de chasse. Une broche fait saillie au-dessus du culot de la cartouche. Le chien, en s'abattant dessus, l'enfonce pour qu'elle percute une amorce interne. Ce premier pas vers le revolver lui vaut un article élogieux dans «the Illustrated London News ». A ses côtés, son fils Eugène Gabriel ne manque pas de S'intéresser aux «stars » du moment les revolvers Colt Navy et Adams. Un an plus tard, Casimir disparait laissant sa veuve dans une situation précaire. Eugène rentre précipitamment de Liège où il effectue un stage d'ajusteur. A 19 ans, il revient avec une idée précise en tête : créer un revolver tirant une cartouche à broche. Il travaille sur cette idée activement, mais il lui faut cent cinquante francs pour déposer son brevet. Sa mère lui refuse cette somme, lasse d'inventions qui rapportent plus de gloire que d'argent ! Cet argent, Eugène va le trouver, en 1854, auprès de son ami Jules Gévelot, fils d'un fabricant de cartouches qui a compris tout l'intérêt du projet. Eugène s'inspire du revolver Colt, mais il améliore son épure pour donner à l'arme une forme plus élégante. Le point fort est sa cartouche en laiton, à broche, qui va se décliner de cinq à quinze millimètres. Sur sa lancée le jeune armurier dépose un brevet pour une arme à double action, en 1855 et un autre en 1860 pour un revolver plus perfectionné. Sa renommée vient de la Marine Impériale qui adopte son arme le 27 octobre 1857. Le modèle réglementaire 1858 sera fabriqué à la Manufacture d'Armes de Saint Etienne, mais notre homme connait un large succès sur les marchés civils et militaire, au point de faire tourner plusieurs ateliers à Liège et à Paris. Le revolver dispose d'une portière dans le bouclier pour introduire les cartouches dans le barillet et d'une baguette sous le canon pour les extraire. Le chargement est rapide, plus besoin de disposer de poudre, amorces et balles !

\subsection{Une nouvelle fabrique}

L'année 1855 marque une étape importante dans la vie de l'inventeur, avec la création d'une société anonyme la «Colt's Patent Fire Arms Manufacturing Company » dont toutes les actions sauf quatre sont détenues par lui-même. Le colonel achève de construire une usine moderne, en pierres 
de taille et en briques, en forme de $\mathrm{H}$ dont la partie centrale est couronnée par un dôme bleu parsemé d'étoile d'or, surmonté d'un poulain cabré (son logo, Colt se traduit par poulain en anglais). Cette fabrique de cent-cinquante mètres de long, en deux étages et demi, est moderne. Elle introduit une division du travail qui précède les principes du taylorisme avec une spécialisation des ateliers et des machines-outils mues par un puissant moteur à vapeur. Les bâtiments administratifs sont reliés à l'usine via une passerelle ouverte. Samuel Colt joue aussi au paternalisme pour loger son millier d'ouvriers dans un ensemble de deux bâtiments. Cependant un panneau, placé en évidence dans l'usine d'Armsmear, comporte ces mots :

«Tout homme employé dans ou pour mon armurerie pour un travail à la pièce ou à la journée, est prié de travailler dix heures pendant le fonctionnement du moteur et quiconque n'y consent pas de bon gré ne doit pas espérer être mon employé !» ${ }^{4}$

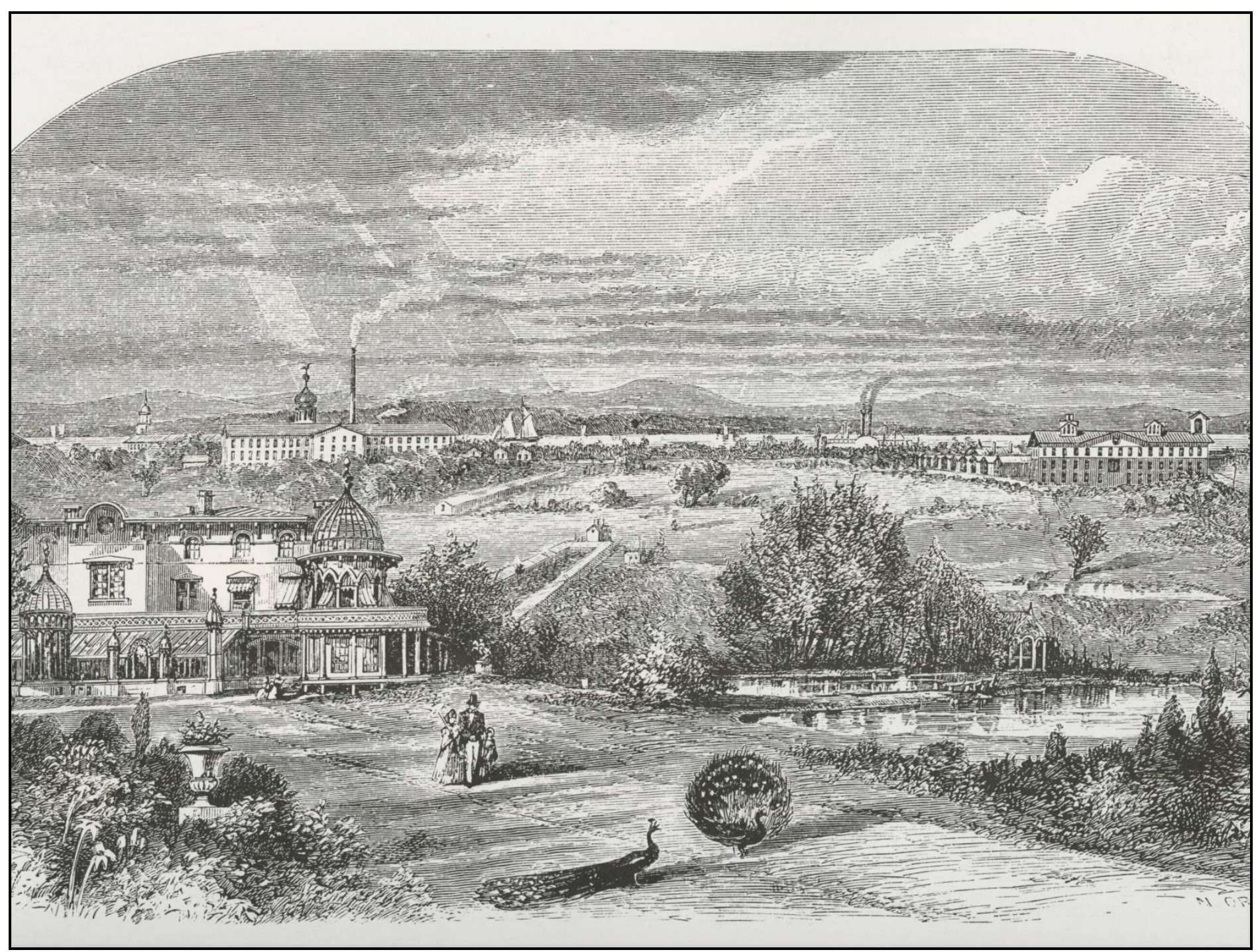

Figure 3. Armsmear en 1857 avec au fond à gauche la fabrique Source : catalogue Armsmear, 1866 (libre de droit).

Cette manufacture devient aussi une sorte d'école technique pour les mécaniciens, les inventeurs, les machinistes et les ajusteurs. On y donne aussi des formations pour les gestionnaires. Ces derniers gèrent les succursales américaines ainsi que les multiples représentants, armuriers locaux ou voyageurs de commerce, un peu partout dans le monde qui représentent la société. L'inventeur Rollin White, détenteur du brevet du barillet foré de part en part pour recevoir des cartouches qui fut

\footnotetext{
${ }^{4}$ Brochure « Armsmear », éditée par la société Colt, 1866. 
à l'origine du succès des revolvers Smith \& Wesson, les machinistes Francis Pratt et Amos Whitney, créateurs de la société de machines-outils qui existe toujours, furent formés chez Colt !

Sam Colt finance aussi un centre socio-éducatif, le Charter Oak Hall, pour éduquer et distraire ses employés. Bibliothèque, école d'art, musique (Colt finance une fanfare), etc., ont pour but de faire évoluer les ouvriers dans l'esprit entrepreneurial américain. Colt préfère former lui-même ses personnels plutôt que de recruter des gens expérimentés. Mêmes les commerciaux sont formés chez lui. Ainsi il les fidélise.

Colt épouse, en juin 1856, Elizabeth Hart Jarvis, fille d'un éminent pasteur de l'église épiscopale et sœur du Vice-président de sa société. Ce mariage est fastueux et les époux partent en voyage de noce six mois en Europe. Naturellement l'entreprenant industriel en profite pour chercher de nouveaux contrats. Il offre ainsi de magnifiques coffrets au Tsar Alexandre II et à ses héritiers. En France, au général Pélissier, qui commandait au siège de Sébastopol, en Scandinavie aux trois Rois de Norvège, Suède et Danemark. De passage à Istamboul, il obtient une commande de cinq mille revolvers auprès du Sultan. Colt s'informe auprès de son ambassade comme auprès des armuriers importateurs locaux pour s'allier les bonnes grâces des gens importants dans chaque pays.

Peu après le retour du couple à Hartford, ils peuvent s'installer à Armsmear, une somptueuse propriété achevée pendant leur absence. Ce palais, situé à côté de l'usine, assure la renommée du colonel avec un parc immense peuplé de paons et de cerfs. Cette année 1855 voit aussi la sortie du revolver issu du brevet de Elisha K Root, avec un chien latéral qui se veut la quintessence de l'arme de poche. Ce petit revolver possède une cage pour le barillet lui donnant une plus grande solidité. Il est aisément dissimulable et intéresse les adeptes d'un port d'arme discret. A partir du mécanisme de son chef d'atelier, Colt développe une gamme de carabines-revolvers. L'arme sera utilisée plus tard par les tireurs d'élite nordistes du colonel Berdan. Mais malgré un matraquage publicitaire la carabine-revolver n'a guère de succès. Il faut dire que le tireur reçoit en plein visage les résidus de poudre et d'amorce...

\subsection{Une nouvelle gamme}

Sam Colt comprend qu'il lui faut renouveler sa gamme car son maitre brevet tombe dans le domaine public en 1857. Il lui est impossible de le prolonger encore une fois. La concurrence se fait rude principalement avec le revolver Remington de 1858 à carcasse fermé plus solide et plus facile à recharger. En 1860, sort le remplaçant du Dragoon, le modèle Army, en 44, aux lignes épurées et au barillet allégé. L'arme tombe à pic, un an avant le début de la guerre de Sécession. Ce revolver va équiper la cavalerie de l'Union. Son succès conduit la firme de Hartford à sortir un second modèle Navy sur le même design, en 1861, naturellement en calibre 36 et un modèle police au barillet cannelé en 1862, lui aussi en calibre 36 mais à cinq coups. Sur sa lancée sort aussi un modèle Pocket Navy en calibre 31. En fait, ce Pocket Navy sert à recycler les pièces des Navy 1851 toujours en stock. A noter que plusieurs revolvers Army 1860 seront achetés par le Gouvernement de la défense nationale, en 1870, en France, pour équiper les armées de la Loire face à l'envahisseur prussien! Les modèles Navy 1861 vont être plébiscités pour leur légèreté. C'est l'arme du général Sheridan (célèbre pour ses charges éclairs et pour avoir ravagé les états confédérés) et de ses cavaliers.

La guerre de Sécession donne un formidable coup d'accélérateur à la production (250 000 revolvers livrés aux Nordistes). Colt axe sa publicité sur «l'acier argent » qui permet de fabriquer un revolver élégant et beaucoup plus léger. Il fait préparer des dossiers de presse lors du lancement de sa nouvelle gamme. Des dossiers très complets et souvent accompagnés d'une arme en cadeau ! De quoi séduire n'importe quel journaliste. Le modèle Army 1860 se décline aussi avec une crosse d'épaulement et d'autres d'exemplaires en coffret sont destinés à être offert. Abraham Lincoln offre ainsi des paires de Colt à ses relations diplomatiques. Sam Colt ne va pas oublier les généraux nordistes et l'US Ordnance (service du matériel) va faire des commandes massives. Très fin et 
élégant le modèle Navy 1861 est prisé pour son calibre réduit. Légers avec leur barillet à cinq coups, les modèles Police et Pocket Navy de 1862 sont les dernières réalisations du colonel [EDW 53].

\subsection{La fin d'une époque}

En 1862, l'usine tourne jours et nuits pour satisfaire les commandes qui proviennent de 1'US Army, la Navy mais aussi des multiples milices et autres régiments privés levés pendant la guerre! Les Sudistes se procurent aussi des revolvers via les Etats dits neutres dans la guerre. Samuel Colt gagne plus d'un million de dollars en 1861 ! La fabrique emploie 1500 personnes en deux équipes de dix heures, mais la santé de Samuel devient chancelante. Epuisement et un rhumatisme articulaire s'ajoutent au stress créer par l'afflux de commandes.

Samuel Colt décède le 10 janvier 1862. Lors de son enterrement, les 1500 employés, portant chacun un brassard de deuil, se dirigent en une lente procession vers la demeure de leur ancien patron pour lui rendre un dernier hommage. Une fois la maison traversée, ils portent le cercueil, en se le passant, jusqu'à sa dernière demeure. Ce bel hommage pour un patron bouleverse tous les officiels. Une plaque est posée sur le centre socio-culturel : "Colt a marqué notre époque de son empreinte, il consacra tous ses efforts à sa magistrale invention, l'œil du monde, et incarna une ère vivante. » [COL 94].

Samuel Colt conscient de son état de santé avait préparé sa succession. Il choisit E.K. Root, son ami d'enfance, qui jouissait de la confiance de madame Colt. Richard Jarvis le frère de cette dernière, continua à assumer la vice-présidence et l'ingénieur en chef Horace Lord assura la direction des ateliers. La société tant sur le plan qualitatif que quantitatif contribua à la cause de 1'Union. Un incendie catastrophique, sans doute un sabotage sudiste, ravage la manufacture le 4 février 1864. Mme Colt fait reconstruire l'usine, y compris la coupole bleue avec un poulain en or sur le dessus. Root meurt en 1865 et Richard Jarvis assure la présidence jusqu'en 1901.

\section{La construction d'une légende}

\subsection{Un personnage hors norme}

Samuel Colt reste un personnage hors norme. C'est un jeune homme aventureux mais très conscient des réalités de son époque. Il connait le poids de la Loi et assure la pérennité de son invention aux Etats-Unis comme en France et en Angleterre. Quand il ne peut pas s'appuyer sur les juridictions de certains pays comme en Belgique, en Russie et en Espagne, il vend ses brevets à des fabricants locaux (par exemple : Sauer und Sohn en Prusse, Jules Lemarche ainsi que Nicolas Gilon à Liège, Alfred Gauvain à Paris, etc.), charge à eux d'en assurer la protection. Dans ce domaine, il va commettre une grosse erreur en refusant d'acheter le brevet de Rollin White, son employé. Daniel Wesson et son complice Horace Smith vont en profiter. Wesson a été à bonne école, les juristes de Colt l'ont mis en faillite en 1849 pour plagiat du brevet Colt sur le revolver Wesson \& Leavitt. Daniel Wesson procédera de même en chargeant Rollin White de poursuivre tous les contrevenants à son maitre brevet (le barillet percé de part en part pour recevoir une cartouche) moyennant un demi dollar par arme fabriquée. La société Smith \& Wesson va devenir un redoutable concurrent à la fin de la guerre de Sécession. La société Colt devra attendre 1873 pour proposer un revolver à cartouche métallique, le célèbre SAA (Single Action Army), magnifié dans tous les westerns [CAS 21].

Colt est un redoutable commercial. On ne compte plus ses cadeaux faits depuis l'époque de Paterson aux officiers et aux dirigeants politiques pour mettre de l'huile dans les contrats. Et ceux dans le monde entier depuis le Roi de Siam en passant par l'émir de Mascate à l'Empereur de Chine. A la grande époque de Hartford, il s'attache les services de graveurs. Il dédicace ses cadeaux « de la part de Samuel Colt », «Par l'inventeur », «avec les compliments du Colonel Colt ». Ces présents somptueux lui ouvrent de nombreuses portes. Il a l'oreille de nombreux officiers du Département 
d'état comme de 1'US Ordnance. Une politique qui va être suivie par ses successeurs. Ainsi le revolver Colt 1873 SAA est préféré au Smith \& Wesson 1870. Pourtant ce dernier est articulé. En soulevant un étrier, l'ensemble canon/barillet bascule vers le bas et en bout de course les douilles sont éjectées. Le Colt lui est rigide et l'on doit charger comme éjecter les cartouches une à une...L US Ordnance rejette le $\mathrm{S} \& \mathrm{~W}$ au motif de trop complexe pour les cavaliers américains ! En 1911, l'US Ordnance va en vain chercher des concurrents contre le pistolet Colt 45 ACP pour les essais. L'américain Savage comme l'allemand Luger vont jeter l'éponge sachant que le marché irait à la société Colt !

\subsection{Un publiciste}

La panoplie des cadeaux Colt comporte la quasi-totalité des modèles de base de la gamme du fabricant. Son préféré est le 1851 Navy dont on recense aujourd'hui le plus grand nombre d'exemplaires. Samuel Colt est devenu une légende américaine surtout grâce à Hollywood. Les accessoiristes préfèrent des revolvers qui puissent tirer des cartouches à blanc plutôt que ceux se chargeant par l'avant du barillet. Leur choix se porte sur le Colt 1873 abondant et avec une silhouette caractéristique, à peu près la même depuis celle des Colt Dragoon. Ils franchissent le pas et ce revolver va devenir celui des cow-boys. On met ce Colt dans tous les films même ceux qui se passent avant 1873 comme pendant la guerre de Sécession (1861-1865). Cette tendance va continuer jusqu'à la fin du XX $\mathrm{XX}^{\mathrm{e}}$ siècle. Il faut dire que les Colt du colonel se chargent par l'avant du barillet. On place le chien en position demi-armé pour assurer la rotation du barillet. Puis on place une charge de poudre, une bourre puis la balle ronde. A l'aide du levier refouloir on enfonce le tout. Le tireur effectue six fois cette opération, puis place les amorces sur les cheminées à l'arrière du barillet. Pas cinématographique tout ça. D'autant plus que les acteurs tirent jusqu'à trente coups sans recharger. Pendant la guerre de Sécession les chiffres nous montrent que sur les 750000 revolvers achetés par le Nord, si les Colt tous modèles confondus arrivent en tête, ils sont talonnés par Remington, Starr et Lefaucheux. Le Remington à carcasse rigide et plus pratique avait les faveurs de beaucoup de soldats. Le Starr à double action (en pressant la détente on assure la rotation du barillet et le départ du coup) alors que ses concurrents sont en simple action, c'est-à-dire qu'il faut armer le chien d'un coup de pouce puis presser la détente, arrive en troisième position. Le revolver français d'Eugène Lefaucheux occupe la $4^{\mathrm{e}}$ place. Il s'agit d'un revolver tirant une cartouche métallique à broche.

Samuel Colt a toujours été un adepte de la publicité. Outre les dossiers de presse, il fait distribuer des milliers de tracts sur ces armes. Il achète aussi des pages dans les journaux et s'assure que des reportages sur ses revolvers comme des témoignages soient publiés au moment où les armées cherchent à se doter de nouvelles armes. Un exemple nous est donné par R. Marcy, en 1859 :

"Tout homme se rendant en territoire indien doit s'armer d'une carabine et d'un revolver qu'il ne doit jamais quitter des yeux... Le revolver Colt est généralement considéré, tant en Europe qu'en Amérique, comme l'arme la plus efficace dans son genre que l'on connaisse actuellement... Pour ma part, je considère la nouvelle carabine Colt comme tout à fait excellente pour assurer la sécurité dans les nouveaux territoires. » [MAR 59].

Toutes ces publicités vont contribuer à sa légende. Surfant sur la mode des « Spaghetti Western », les armuriers italiens Uberti, Pietta, etc vont fabriquer des répliques des revolvers Colt. Ces armes vont connaitre un énorme succès au point que des compétitions de tir leurs sont dédiées. En vente libre car obsolètes, elles vont enrichir la légende Colt [CAS 21].

Samuel Colt est un meneur d'hommes. Il offre logements, promotions, formations et distractions à ses ouvriers et sait par-là se les attacher. Cet attachement se verra lors de ses obsèques. Mais là encore il s'git d'un investissement pour la bonne marche de son entreprise ! Notre homme sait cultiver son personnage. Il a du flair en faisant évoluer son invention pour qu'elle corresponde à la 
fois aux besoins comme aux goûts de ses clients. Sa devise résume le personnage : «Dieu a fait des hommes grands et d'autres petits, je les ai rendus égaux. »

\section{Conclusion}

Les éléments du succès de Samuel Colt sont multiples. En premier lieu les qualités techniques de son invention. Mais aussi ses protections juridiques pour ses brevets, l'humeur du temps (conquête de l'Ouest, guerre avec le Mexique puis guerre de Sécession), la productivité de sa fabrique, ainsi que sa clairvoyance publicitaire et promotionnelle. Les armes offertes par Samuel Colt ont joué un grand rôle. C'est en partie à cause de cette pratique que la société Colt est plus qu'une entreprise, une institution américaine du $\mathrm{XIX}^{\mathrm{e}}$ au $\mathrm{XX}^{\mathrm{e}}$ siècles.

Les exemplaires des cadeaux de Samuel Colt, politique poursuivie par ses successeurs, offrent un large champ d'investigation aux collectionneurs dans le domaine des armes anciennes. De leur point de vue, aucun autre fabricant d'armes n'arrive à sa hauteur hormis Nicolas Noël Boutet, à moindre échelle, l'arquebusier de l'Empereur Napoléon $\mathrm{I}^{\mathrm{er}}$ ! Samuel Colt est un véritable entrepreneur et un innovateur. Il a compris l'importance des aspects juridiques, celle de la publicité, celle de la rationalité de ses fabriques comme celle du bien-être de ses ouvriers pour en obtenir un rendement supérieur.

\section{Bibliographie}

[IVE 10] BERNARD H., Mme Samuel Colt, Benifield Publishing, Hartford, 1866, réédition 1876.

[CAD 95] CADIOU Y., Les Colt, éditions du Portail, La Tour du Pin, 1995.

[CAS 14] CASANOVA D., Histoire du Revolver, ETAI, Anthony, 2014.

[CAS 21] CASANOVA D., Les armes au cinéma, Editions du Plateau, Aix en Provence, 2021.

[COL 94] COLT Mme, A Memorial of Caldwell Hart Colt, Colt Hartford 1894.

[EDW 53] EDWARDS W, Civil war guns, Stackpole Company, Harrisburg 1953.

[HAV 40] HAVEN \& BELDEN, A history of the Colt Revolver, Morrow \& Co, New York 1940.

[IVE 10] IVEY D., A registry \& history texas rangers, Mc Farland, 2010.

[KIH 59] KIHN P., « Colt in Hartford », the Connecticut Historical Society Bulletin, Hartford, 1959.

[MAR 11] MARCOT R., PAXTON R., « Colt Brevete Revolvers » Northwood Heritage Press, Tucson, 2011.

[MAR 59] MARCY R., The Prairie Traveler. A hand-book for overland expeditions, with maps, illustrations, and itineraries of the principal routes between the Mississippi and the Pacific, Harper, New-York, 1859.

[SEL 71] SELLERS F., Museum Restoration Service, Ottawa, 1971.

[SIB 78] SIBLEY M. M., Samuel H. Walker's Account of the Mier Expedition, Austin, 1978.

[WIL 88] WILSON R.L. Colt une légende américaine, EPA, Paris, 1988 